\title{
pH-Responsive PLGA Nanoparticle for Controlled Payload Delivery of Diclofenac Sodium
}

\author{
Shalil Khanal ${ }^{1,2}$, Udhab Adhikari ${ }^{2,3}$, Nava P. Rijal ${ }^{2,4}$, Shanta R. Bhattarai ${ }^{5}$, \\ Jagannathan Sankar ${ }^{2}$ and Narayan Bhattarai ${ }^{2,4, *}$ \\ 1 Department of Energy and Environmental Systems, North Carolina A \& T State University, Greensboro, \\ NC 27411, USA; skhanal@aggies.ncat.edu \\ 2 Engineering Research Center Revolutionized Metallic Biomaterials, North Carolina A \& T State University, \\ Greensboro, NC 27411, USA; uadhikar@aggies.ncat.edu (U.A.); nprijal@aggies.ncat.edu (N.P.R.); \\ sankar@ncat.edu (J.S.) \\ 3 Department of Mechanical Engineering, North Carolina A \& T State University, Greensboro, NC 27411, USA \\ 4 Department of Chemical, Biological, and Bioengineering, North Carolina A \& T State University, \\ Greensboro, NC 27411, USA \\ 5 Department of Experimental Radiation Oncology, University of Texas MD Anderson Cancer Center, \\ Houston, TX 77030, USA; sbhattarai@mdanderson.org \\ * Correspondence: nbhattar@ncat.edu; Tel.: +1-336-285-3652
}

Academic Editor: Dusan Losic

Received: 13 June 2016; Accepted: 28 July 2016; Published: 2 August 2016

\begin{abstract}
Poly(lactic-co-glycolic acid) (PLGA) based nanoparticles have gained increasing attention in delivery applications due to their capability for controlled drug release characteristics, biocompatibility, and tunable mechanical, as well as degradation, properties. However, thorough study is always required while evaluating potential toxicity of the particles from dose dumping, inconsistent release and drug-polymer interactions. In this research, we developed PLGA nanoparticles modified by chitosan (CS), a cationic and $\mathrm{pH}$ responsive polysaccharide that bears repetitive amine groups in its backbone. We used a model drug, diclofenac sodium (DS), a nonsteroidal anti-inflammatory drug (NSAID), to study the drug loading and release characteristics. PLGA nanoparticles were synthesized by double-emulsion solvent evaporation technique. The nanoparticles were evaluated based on their particle size, surface charge, entrapment efficacy, and effect of $\mathrm{pH}$ in drug release profile. About $390-420 \mathrm{~nm}$ of average diameters and uniform morphology of the particles were confirmed by scanning electron microscope (SEM) imaging and dynamic light scattering (DLS) measurement. Chitosan coating over PLGA surface was confirmed by FTIR and DLS. Drug entrapment efficacy was up to $52 \%$. Chitosan coated PLGA showed a $\mathrm{pH}$ responsive drug release in in vitro. The release was about $45 \%$ more at $\mathrm{pH} 5.5$ than at $\mathrm{pH} 7.4$. The results of our study indicated the development of chitosan coating over PLGA nanoparticle for $\mathrm{pH}$ dependent controlled release DS drug for therapeutic applications.
\end{abstract}

Keywords: nanoparticle; PLGA; chitosan; diclofenac drug; drug delivery; $\mathrm{pH}$ dependent

\section{Introduction}

Drug delivery devices using biodegradable and biocompatible poly(lactic-co-glycolic acid) (PLGA) based nanoparticles have attracted a great deal of attention, as these systems can provide a sustained and controlled drug release and reduce side effects [1-5]. They can protect drugs from degradation and enhance their stability. Moreover, due to their size, nanoparticles can penetrate specific tissues via (i) the fenestrations present in the endothelium of cancer and inflamed tissue or (ii) via receptors over expressed by target cells [6]. This allows a specific delivery of the loaded drugs, proteins, peptides or 
nucleic acids to their target tissue [7]. Another major advantage of PLGA over other polymers is that PLGA is approved by the FDA.

Nonsteroidal anti-inflammatory drugs (NSAIDs) are one of the most commonly prescribed drugs in the world [8]. Analgesic and anti-inflammatory effects are exerted by NSAIDs through the inhibition of the cyclooxygenase family of enzymes. A local delivery of anti-inflammatory drugs could be an interesting approach to avoid potential side effects resulting from systemic applications [9]. Diclofenac Sodium (DS) is a NSAID that is indicated in relief of signs and symptoms of ankylosing spondylitis, rheumatoid arthritis, osteoarthritis, migraines and menstrual pain. Diclofenac has been frequently used to treat moderate post-operative or post-traumatic pain. However, the short biological half-life of DS severally reduces its application for the long-term treatment of inflammatory disorders. Long-term continuous infusion or multiple dosing schedules like other NSAIDs, DS also has common side effects like gastrointestinal lesion formation, and renal damage [10]. Several research strategies have been focused to overcome these limitations, when DS drug encapsulated into polymer-based nanoparticles are administered orally [11-13]. Biodegradable polymer based nanoparticle; particularly PLGA/drug formulation strategies, can greatly enhance the therapeutic drug concentration in the blood stream for a long period of time and can subsequently reduce the multiple drug dose schedules. However, it is difficult to achieve functionalities, such as active targeting in PLGA-based drug delivery systems, due to deficiency of functional groups on PLGA surface. The negative charge on PLGA surface reduces cell affinity and immobilization of cell-targeting molecules. Therefore, surface modification of PLGA-based drug carriers is necessary for getting best ability of the system $[4,14]$. Cationic surface modifications of such systems has been accomplished by using cetyltrimethylammonium bromide, polyethyleneimine, poly(2-dimethylamino)ethyl methacrylate, didodecyldimethylammonium bromide, poly (ethylene glycol) and chitosan $[4,15,16]$.

Chitosan (CS) is a cationic and $\mathrm{pH}$ responsive polysaccharide produced by deacetylation of chitin. It is a structural element in the exoskeleton of crustaceans and insects [17]. The presence of primary amino groups in chitosan is responsible for several important properties such as in situ gelation, transfection, permeation enhancement, mucoadhesion, and efflux pump inhibitory. Chitosan has been widely used in the field of surface modification due to several desirable properties which includes-mild processing conditions, chemical reactivity, minimal foreign body reaction, and cost effectiveness [18-20]. By modifying the surface of PLGA NPs, CS provides the following advantages: (i) a decreased burst effect in encapsulated drug release; (ii) increased stability of macromolecules such as proteins; (iii) enhances the Zeta potential inversion, and promotes cellular adhesion and retention of the delivery system at the target site; and (iv) offers the possibility of conjugating targeting ligands to free amino groups on its surface [21-24]. Chitosan and its derivative also help in the $\mathrm{pH}$ dependent release of drug in different nanoparticle systems [25-28]. Chitosan modified nanoparticles has been used for drug delivery where it could play dual function for protecting the DS drug against the action of the enzymes and gastric fluids, and reduction of the gastrointestinal irritation caused by DS drugs through $\mathrm{pH}$ dependent controlled release system from the PLGA particles.

In this work, we investigated the ability of CS coated PLGA based NPs to entrap and release, a model drug, NSAID diclofenac, in a controlled manner. We synthesized core-shell NPs in which PLGA being core and CS being an external shell on NPs surface. Double-emulsion solvent evaporation technique was used to synthesize CS coated DS loaded nanoparticles (CS-DS-PLGA). Uncoated nanoparticles of PLGA (DS-PLGA) were used as control for comparative study. The physio-chemical and surface properties of the different formulations of CS-DS-PLGA and DS-PLGA were studied. In vitro drug release profiles were evaluated at different $\mathrm{pH}$. 


\section{Results and Discussions}

\subsection{Design and Characterization of PLGA Nanoparticles}

PLGA particles can be prepared by single- or double-emulsion techniques. These methods provide the ability to customize various particle characteristics including size, encapsulant, and surface properties [29]. In this study, we fabricated the nanoparticles by double emulsion solvent evaporation technique. The schematic representation of the process is shown in Figure 1. Surface morphology and size distribution of prepared nanoparticles were examined through SEM images. Figure 2 shows the surface morphology and size distribution of corresponding nanoparticle. The microspheres were found to be uniform spherical, and have smooth surfaces with some deviations and nominal aggregation. From size distribution study using ImageJ software, the mean diameters of DS-PLGA and CS-DS-PLGA nanoparticles were found as 398.8 and $403.8 \mathrm{~nm}$, respectively. The results obtained from the DLS measurement is shown in Table 1 . These results are in agreement in size with the scanning electron microscopic images (Figure 2). Measurements of the particle size showed a slight increase in the diameter (from $415 \pm 6 \mathrm{~nm}$ to $420 \pm 6 \mathrm{~nm}$ ) by the addition of chitosan. It is found that the chitosan uncoated PLGA nanoparticles have a zeta potential of $-14 \pm 0.4 \mathrm{mV}$, which is due to the presence of carboxyl end groups on nanoparticle surfaces. Obviously, after the chitosan treatments, sample shows a positive zeta potential $(27 \pm 0.6 \mathrm{mV})$, which indicates the amine groups present in the chitosan successfully coated onto the PLGA nanoparticle surfaces.

Particles of PLGA, smaller than $10 \mu \mathrm{m}$ and coated with chitosan, have great potential applications in ocular drug delivery. These particles exhibit reduced eye irritation and enhanced corneal penetration with better drug bioavailability compared to larger PLGA particles ( $>10 \mu \mathrm{m})$ [30-32]. Additionally, positively charged nanoparticles show prolonged retention times on the corneal surface when compared to negatively charged nanoparticles because of possible electrostatic interactions between the positively charged particles with negatively charged mucin in eyes [30,32].

The FTIR spectra confirmed the chitosan adsorption to the surface of PLGA. Figure 3 shows the FTIR spectra of PLGA, CS, DS, DS-PLGA and CS-DS-PLGA. In the PLGA spectrum, the peak of C-O-C stretching is at $1175 \mathrm{~cm}^{-1}, \mathrm{C}-\mathrm{H}$ bend is at $1390-1440 \mathrm{~cm}^{-1}, \mathrm{C}=\mathrm{O}$ is at $1760 \mathrm{~cm}^{-1}$ and $\mathrm{C}-\mathrm{H}$ stretch is at $3000 \mathrm{~cm}^{-1}$. From CS-DS-PLGA spectra, the chitosan adsorption to the surface of PLGA was confirmed by two intense peaks at 1545 and $1630 \mathrm{~cm}^{-1}$, corresponding to amine and amide bonds, and broad $\mathrm{N}-\mathrm{H}$ signal of chitosan at $3200-3700 \mathrm{~cm}^{-1}$. Amino peak (N-H stretching), $-\mathrm{C}=\mathrm{O}$ stretching of the carboxyl ion and $\mathrm{C}-\mathrm{Cl}$ stretching appeared at 3320,1573 and $768 \mathrm{~cm}^{-1}$, respectively, representing DS in PLGA sample.

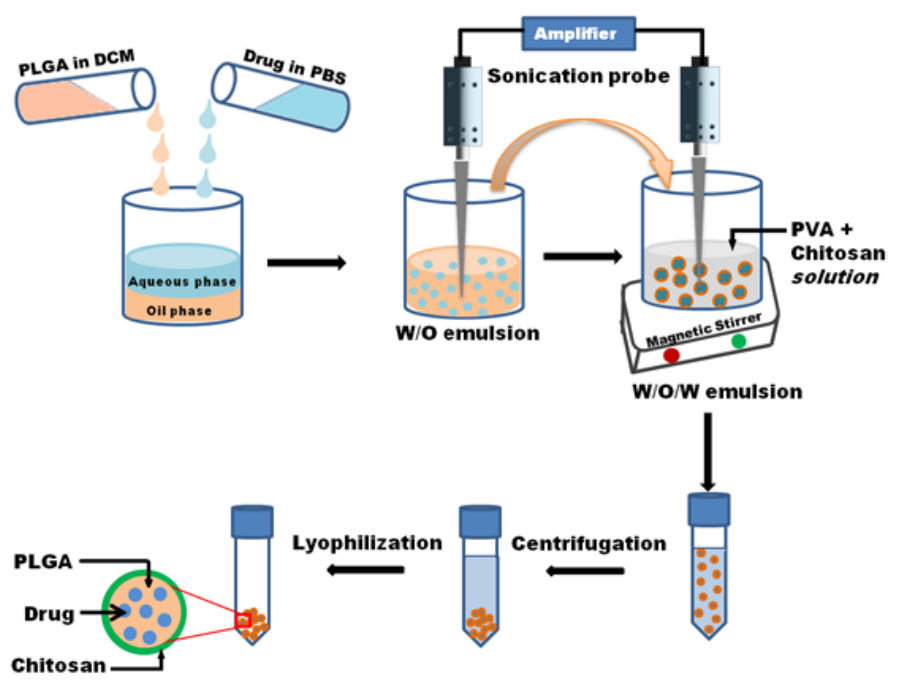

Figure 1. Schematic representation of nanoparticle fabrication. 


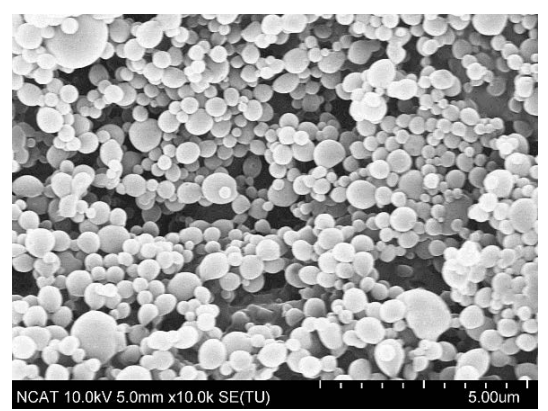

(A)

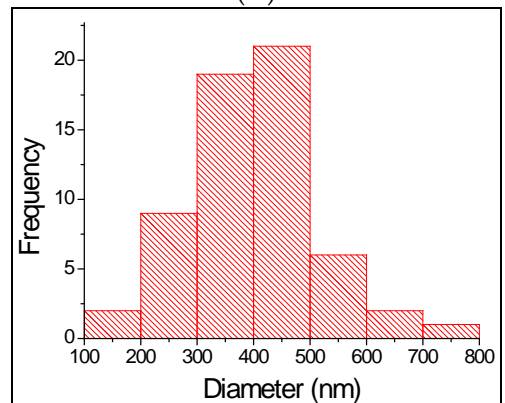

(C)

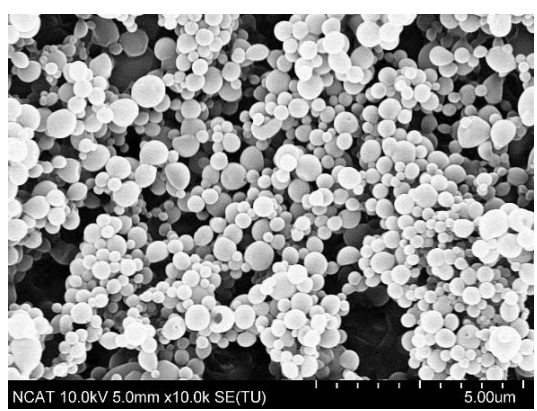

(B)

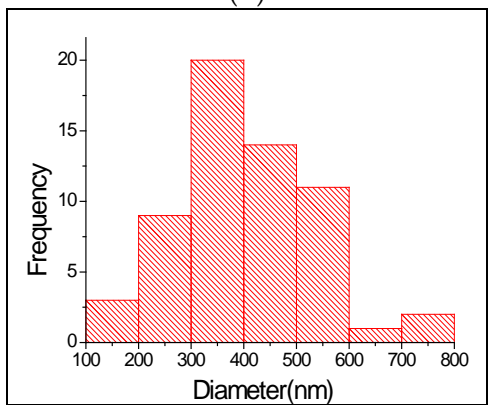

(D)

Figure 2. SEM images (A,B) and size distribution $(\mathbf{C}, \mathbf{D})$ where, $(A, C)$ DS-PLGA; (B,D) CS-DS-PLGA. The microspheres were found to be uniform with some deviations and nominal aggregation.

Table 1. Size distribution and Zeta potential of NPs.

\begin{tabular}{cccc}
\hline Sample & Size $(\mathbf{n m}) \mathbf{( M e a n} \pm$ S.D) & Zeta Potential $(\mathbf{m V})($ Mean \pm S.D) & Drug Contain $(\%)$ \\
\hline DS-PLGA & $415 \pm 6$ & $-14 \pm 0.4$ & 3.92 \\
CS-DS-PLGA & $420 \pm 6$ & $27 \pm 0.6$ & 3.13 \\
\hline
\end{tabular}

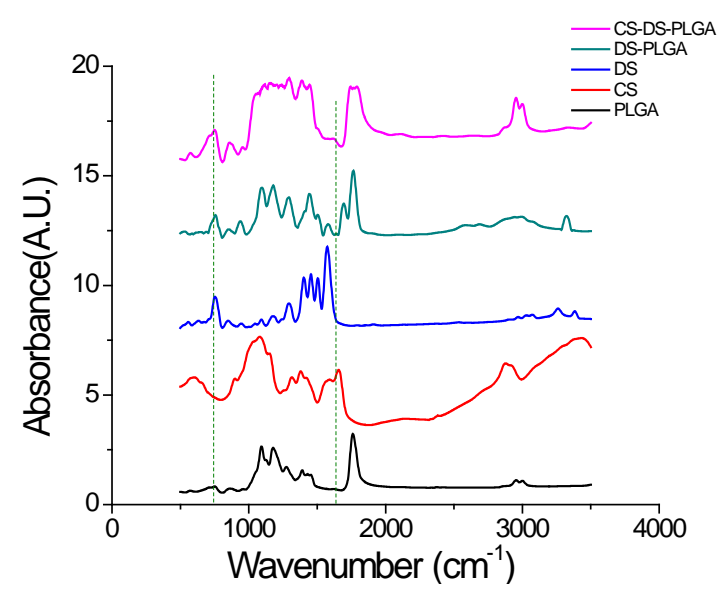

Figure 3. FTIR spectra of PLGA, CS, DS, DS-PLGA, and CS-DS-PLGA. The major peaks for all nanoparticles samples is measured at $1175 \mathrm{~cm}^{-1}$ and $1760 \mathrm{~cm}^{-1}$ which corresponds to the standard basic measurement of PLGA absorption band. Characteristic absorption peak of chitosan at $1545 \mathrm{~cm}^{-1}$ and $1630 \mathrm{~cm}^{-1}$ and that of drug at $768 \mathrm{~cm}^{-1}$ confirms their presence in respective nanoparticles.

The XRD patterns for PLGA, chitosan (CS), diclofenac sodium (DS), DS-PLGA and CS-DS-PLGA are shown in Figure 4. For PLGA and CS, the XRD pattern showed no distinct peak, a fact that indicates, these polymers are amorphous $[33,34]$. DS powder exhibited crystalline structure with distinct XRD peaks appearing at $14.90,20.74,25.96,26.82$ and 27.70 at $2 \theta$ values [35]. In the case of the nanoparticles, 
the principal peaks of the drug existed but could be observed with less intensity, reflecting that the DS existed mostly in an amorphous state and was successfully entrapped into the NPs.

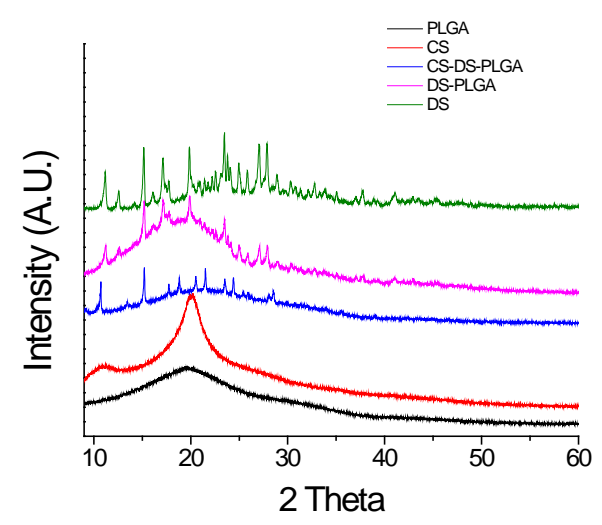

Figure 4. XRD spectra of PLGA, CS, DS, DS-PLGA, and CS-DS-PLGA. The characteristic diffraction peaks of drug at $14.90,20.74,25.96,26.82$ and 27.70 at $2 \theta$ values are clearly observed in DS-PLGA and CS-DS-PLGA nanoparticles. PLGA nanoparticles and chitosan powder showed no distinct peak which indicates the amorphous nature of these polymers.

The relative amount of diclofenac sodium (as compared with the amount of the polymer) was varied between $3 \%$ and $12 \%(\mathrm{wt} / \mathrm{wt}$ ) to study effect of proportional amount of drug substance on the entrapment efficiency. The result (Figure 5) shows that the entrapment efficiency was increased from $40 \%$ to $52 \%$ with increased drug amount from $3 \%$ to $6 \%(\mathrm{wt} / \mathrm{wt}$ ) but, after that there is no significance difference for increased drug amount. Thus, $6 \%$ drug was used as optimized condition for further study. The encapsulation efficiencies were low because of the leakage of DS to the external medium, resulting in decreased drug content in the nanoparticles. The result for drug calculation from optimized samples is shown in Table 1. The amount of entrapped DS in PLGA NPs was measured before and after CS coating, which was not significant $(p>0.05)$ for both samples, which suggested that there was no drug leakage during the coating procedure.

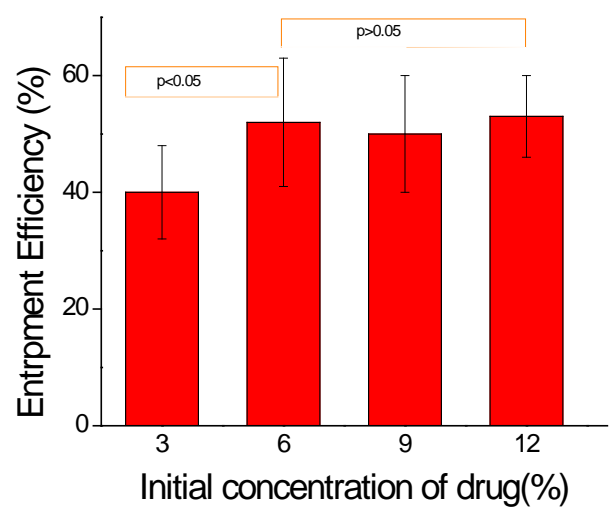

Figure 5. Drug entrapment efficiency of CS-DS-PLGA nanoparticle. Optimum drug entrapment efficiency was found to be $52 \%$ with $6 \%$ initial drug. After that, there is no significance difference with increasing initial drug concentration (mean \pm S.D., $n=3$ ).

\subsection{Effect of Chitosan Concentration}

The drug release was studied for 7 days from chitosan coated sample, prepared with different concentration to study the effect of concentration change in chitosan. The obtained result is shown in Figure 6 . Though all sample showed a burst release of drug initially, the sample with $0.4 \%$ chitosan had higher release. However, the overall drug release pattern is similar for all three samples for 7 days 
with no significant difference. Thus, for further release study, we used the sample from $0.4 \%$ chitosan. The overall drug release depends on various factors, such as molecular weight of polymer, amount of drug entrapped, size and overall porosity of the particles. For applications requiring higher release of encapsulated drugs, low molecular weight PLGA can be used, which forms highly porous surface for greater drug release [36].

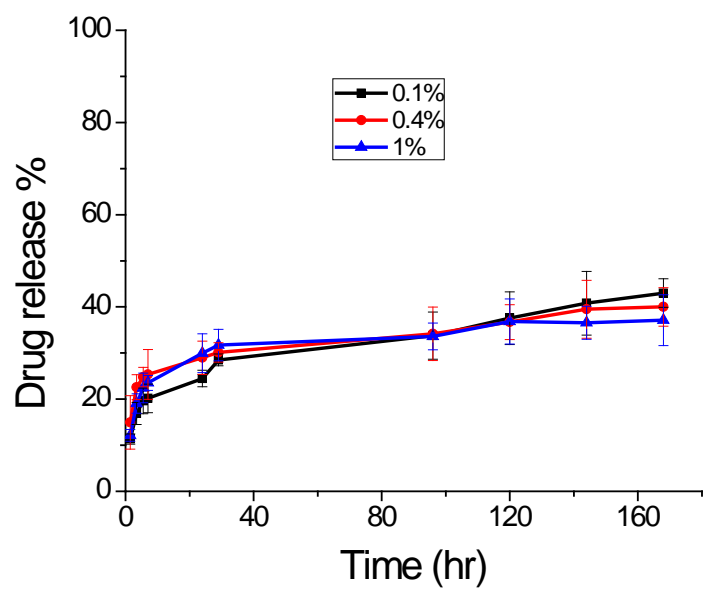

Figure 6. Drug release profile with variable chitosan concentration at $\mathrm{pH} 7.4$ and $37^{\circ} \mathrm{C}$ (mean \pm S.D., $n=3$ ).

\subsection{In Vitro Drug Release}

In vitro drug release profiles of DS from the treated CS-DS-PLGA nanoparticles and untreated DS-PLGA nanoparticles are shown in Figure 7. Both samples show a burst release in the first $8 \mathrm{~h}$. The modified CS-DS-PLGA nanoparticles exhibited heavier burst release than the untreated nanoparticles because the chitosan and DS have opposite surface charge and some of the drug DS is adsorbed to PLGA nanoparticle surface during the synthesis and solvent evaporation process. Swelling of PLGA polymer, pore diffusion, polymer erosion and degradation contribute to the drug release from both samples [37]. Therefore, after the burst release, both nanoparticles show moderate and sustained release. Chitosan is more hydrophilic than PLGA, which allows PBS solution to more easily penetrate into the nanoparticle matrix and release more drug molecules during the same time. In addition, the presence of positively charged chitosan on the surface of nanoparticles attracts the negatively charged DS drug towards it, thereby facilitating a faster release. Both of these factors contribute to a higher cumulative drug release from PLGA-CS nanoparticles [4].

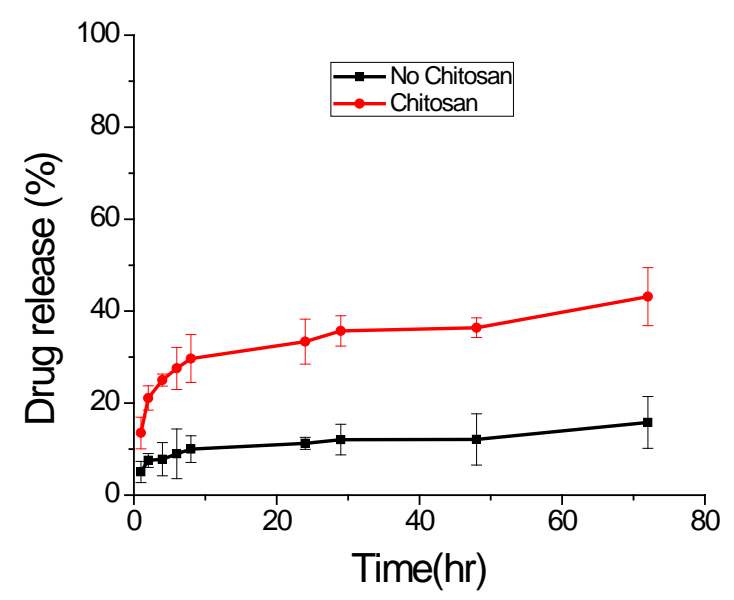

Figure 7. In vitro drug release at $\mathrm{pH} 7.4$ and $37^{\circ} \mathrm{C}$. The chitosan coated sample was prepared with $0.4 \%$ chitosan (mean \pm S.D., $n=3$ ). 


\subsection{Effect of $\mathrm{pH}$ on Drug Release}

The $\mathrm{pH}$ dependent in vitro drug release from nanoparticles was carried out in two different buffered solutions with $\mathrm{pH} 7.4$ and 5.5 at $37^{\circ} \mathrm{C}$. The in vitro release profile of DS from different NPs is presented in Figure 8. It shows biphasic character with a brisk initial burst release followed by a sustained release. The graph in Figure 8a shows the release profiles obtained for chitosan uncoated PLGA NPs. The release pattern is similar, but only about $20 \%$ release in 7 days for both $\mathrm{pH}$ values, which suggests $\mathrm{pH}$ independent nature of this sample. As can be seen, both release profiles exhibited rapid burst effect during the first $8 \mathrm{~h}$, indicating no chemical interactions between DS and the polymeric chains [21]. However, in case of chitosan-coated PLGA nanoparticles, the release profile is highly different, as shown in Figure 8b. For pH 5.5, there is nearly 90\% release of drug, but the same sample the release is only about $43 \%$ at $\mathrm{pH} 7.4$ within 7 days. This result indicates the $\mathrm{pH}$ dependent nature of chitosan coated sample as expected due to higher solubility of chitosan in acidic $\mathrm{pH}$. Chitosan has a primary amine group in its glucosamine monomeric unit with low $\mathrm{pKa}$ value (6.3), which is protonated and positively charged at low $\mathrm{pH}$, making chitosan soluble in aqueous solution. At higher $\mathrm{pH}$ (alkaline region), this amine group becomes deprotonated and uncharged, hence making insoluble biopolymeric hydrogel networks.

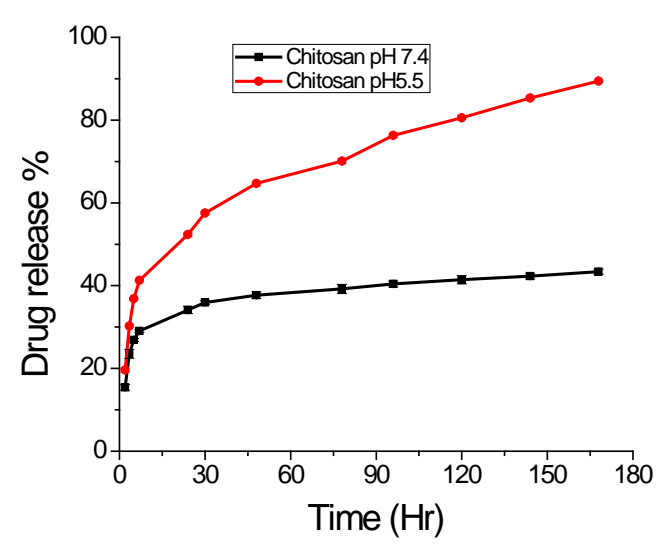

(a)

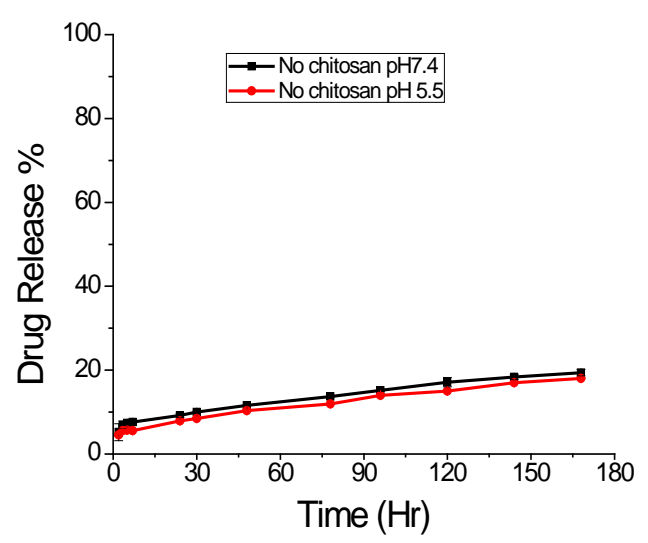

(b)

Figure 8. Effect of different $\mathrm{pH}$ on drug release at $37^{\circ} \mathrm{C}$. (a) Chitosan coated; (b) Chitosan uncoated (mean \pm S.D., $n=3$ ).

\section{Materials and Methods}

\subsection{Materials}

Poly(lactic-co-glycolic acid) (PLGA, lactic acid/glycolic acid = 50:50, with ester end groups, inherent viscosity $(0.26-0.54 \mathrm{dL} / \mathrm{g}))$ was purchased from Durect Corporation (Birmingham, AL, USA). Chitosan (medium Mw), poly (vinyl alcohol) (PVA, 99\% hydrolyzed), diclofenac sodium drug was purchased from Sigma Aldrich (St. Louis, MO, USA). Dichloromethane (DCM, 99\%), glacial acetic acid and sodium hydroxide ( $\mathrm{NaOH}$ ) were purchased from Acros Organics (Morris Plains, NJ, USA). Phosphate buffered saline (PBS, $1 \times$ solution) and acetate buffer ( $\mathrm{pH}$ 5.5) were purchased from Thermo Fisher Scientific (Fair Lawn, NJ, USA).

\subsection{Nanoparticle Preparation}

PLGA nanoparticles (NP) were prepared by a single/double emulsion solvent evaporation technique with some modifications [38]. Briefly, $200 \mathrm{mg}$ of PLGA was dissolved in $2 \mathrm{~mL}$ of DCM and added drop wise to $5 \mathrm{~mL}$ of $0.3 \%$ PVA solution under constant vortex. The solution was emulsified in ice bath for 2 min by using Ultra-Sonicator (Thermo Fisher Scientific, Fair Lawn, NJ, USA) at 40\% amplitude with $10 \mathrm{~s}$ on/off pulse mode. Then, the emulsified solution was transferred into $45 \mathrm{~mL}$ of 
$0.3 \%$ PVA solution and the mixture was stirred for $3 \mathrm{~h}$ to allow the solvent evaporation and particle hardening. The particles were collected by Sorvall Stratos Centrifuge (Thermo Fisher Scientific, Fair Lawn, NJ, USA) at $10016 \times \mathrm{g}$ for $30 \mathrm{~min}$ at $4{ }^{\circ} \mathrm{C}$ and washed three times with DI water. The purified PLGA NPs were lyophilized for 2 days by using Freezone Freeze Dryer (Labconco, Kansas City, MO, USA) and stored at $-20^{\circ} \mathrm{C}$.

For drug encapsulation, the diclofenac drug solution was mixed with polymer solution and the mixture was sonicated for $30 \mathrm{~s}$ before mixing with PVA emulsifier as above. The chitosan coated samples were prepared by mixing the chitosan solution (in 1\% Acetic acid) with PVA solution before adding the emulsified polymer solution for stirring.

\subsection{Morphology Study}

The surface morphology of the nanoparticles was observed by scanning electron microscope (SEM) (Hitachi SU8000, Hitachi High-Technologies Corporation, Tokyo, Japan). Specimens were attached to the sample holder by using double sided carbon tape. Prior to imaging with the SEM, samples were sputter coated with gold under vacuum by using Polaron SEM Coating System (Quorum Technologies, East Sussex, UK) for $2 \mathrm{~min}$. The images were acquired at an accelerating voltage of $10 \mathrm{kV}$ and current of $5 \mu \mathrm{A}$.

The size distribution of the nanoparticles was analyzed through SEM images with the use of ImageJ software (NIH, Bethesda, MD, USA). Physical length was converted to pixels with the help of scale bar. Seventy-five nanoparticles of each group $(n=3)$ were measured in pixels. Average size and standard deviation were calculated based on converted ImageJ data.

\subsection{Size Distribution and Zeta Potential}

The NPs size and zeta potential were measured by using dynamic light scattering technique (DLS) on Zetasizer Nano ZS (Malvern Instruments, Malvern, UK). The freshly prepared and purified suspension of NPs was diluted in deionized (DI) water and the mixture was vortexed for $2 \mathrm{~min}$. The analysis was carried out at a scattering angle of $90^{\circ}$ and a temperature of $298 \mathrm{~K}$. The Z-average diameter and zeta potential were calculated using Malvern software. Zeta potential, based on the electrophoretic mobility was measured to estimate the surface charge. For statistical analyses, three different readings were taken.

\subsection{Fourier Transform Infrared (FTIR) Analysis}

FTIR was used to analyze the chemical interactions among PLGA, chitosan and DS. Absorbance spectra were recorded at 200 scans using IR Prestige21 FTIR Spectrometer (Shimadzu, Kyoto, Japan) from wavenumbers of 400 to $4000 \mathrm{~cm}^{-1}$ with a resolution of $4 \mathrm{~cm}^{-1}$. A pellet was made for FTIR measurement by dispersing the nanoparticles in $\mathrm{KBr}$ matrix and compressing at high pressure.

\subsection{X-Ray Diffraction (XRD) Study}

Crystallography and phases of different nanoparticles were examined by using a Bruker AXS D8 Discover X-ray diffractometer (Bruker Daltonics Inc., Billerica, MA, USA) with $\mathrm{Cu}-\mathrm{K} \alpha$ radiation. The $X$-ray diffraction experiments were performed using a locked-coupled scan with a scanning range

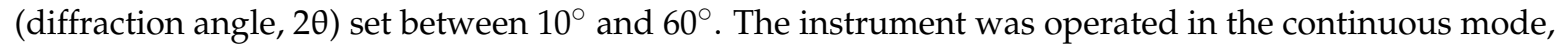
in increments of $0.0146^{\circ}, 2 \theta$. All experiments were performed at room temperature.

\subsection{Entrapment Efficiency and Drug Content}

The entrapment efficiency and drug content of DS loaded nanoparticles was analyzed according to previous methods [36,39]. Briefly, $50 \mathrm{mg}$ of NPs containing drug were dissolved by adding $5 \mathrm{~mL}$ of DCM and the polymer was precipitated after the addition of methanol. During the synthesis of nanoparticles, the amount of drug in the supernatant after each step of centrifugation was quantified. 
A calibration curve was developed using the known standard concentrations of DS dissolved in DI water. The spectrophotometric quantification was achieved by taking the absorbance at $277 \mathrm{~nm}$. The encapsulation efficiency was calculated using the formula:

$$
E E \%=\frac{\text { Total amount of drug used }- \text { amount of drug in the supernatant }}{\text { Total amount of drug used }} \times 100
$$

Then, percentage of drug content was calculated using the formula:

$$
\text { Experimental drug loading }=\frac{\mathrm{L}}{\mathrm{Lo}} \times 100
$$

where $\mathrm{L}$ is the actual drug content and Lo is the weighed quantity of beads.

\subsection{Study of Effect of Chitosan Concentration on Drug Release}

Three different samples were prepared by using different concentration $(0.1 \%, 0.4 \%$ and $1 \%)$ of chitosan by same technique as described above. In order to study, $60 \mathrm{mg}$ of each sample was added to $3 \mathrm{~mL}$ of 1X PBS solution and incubated in a Shaking Incubator (Dubnoff Shakebath-2876, Thermo Fisher Scientific, Fair Lawn, NJ, USA) at $37^{\circ} \mathrm{C}$ and $50 \mathrm{rpm}$. At different time intervals, aliquots of $2 \mathrm{~mL}$ were collected from each sample solution and added same volume of fresh PBS to keep the final volume constant throughout the study. Then, released drug concentration was calculated by using a UV-Spectrophotometer (GENESYS 10S, Thermo Fisher Scientific, Fair Lawn, NJ, USA) at a wavelength of $277 \mathrm{~nm}$. Since there is no significant difference in drug release from sample with different chitosan concentration a further test was performed with a sample prepared by using $0.4 \%$ chitosan.

\subsection{In Vitro Drug Release Study}

In order to investigate the release of Diclofenac from DS-PLGA and CS-DS-PLGA NPs, a fixed amount $(50 \mathrm{mg}$ ) of each formulation was incubated in $6 \mathrm{ml}$ of $1 \times$ phosphate buffer solution (PBS, $\mathrm{pH} 7.4)$ at $37^{\circ} \mathrm{C}$ and $50 \mathrm{rpm}$. At fixed time intervals, $5 \mathrm{~mL}$ of the supernatant was withdrawn and replaced with fresh buffer. The absorbance of each solution was measured at $277 \mathrm{~nm}$ and compared with a calibration curve to determine the amount of drug released. All experiments were performed in triplicate.

\subsection{Determination of Effect of $p H$ on Drug Release}

Two different buffer solutions: 1X PBS (pH 7.4) and Acetate Buffer ( $\mathrm{pH}$ 5.5) were used to study the effect of $\mathrm{pH}$ on drug release. Additionally, the release study was performed with a similar method as that described in Section 3.8.

\subsection{Statistical Analysis}

All tabulated results were expressed as mean \pm S.D. Data were analyzed for significance with OriginPro software (Origin Lab, Northampton, MA, USA) using one-way analysis of variance (ANOVA). Post hoc Tukey's test was performed with ANOVA for multiple comparisons. The $\alpha$-value was set to 0.05 and $p$-values less than 0.05 were considered statistically significant.

\section{Conclusions}

Chitosan coated NPs were successfully synthesized by physical adsorption of its polymeric chains on the surface of drug-loaded PLGA polymeric particles. A physical approach for studying DS release from polymeric NPs (average diameter $390-420 \mathrm{~nm}$ ), has been proposed. CS coating influenced higher release of DS and $\mathrm{pH}$ dependent behavior for PLGA nanoparticles. This release profile could be of potential interest for drug delivery in in vivo human therapies, by producing a constant and uniform concentration of drug inside cells with their long-term biochemical effects. The results of our study 
indicate the development of chitosan coating over PLGA nanoparticle for $\mathrm{pH}$ dependent controlled release DS drug for therapeutic applications.

Acknowledgments: This work is supported financially by the National Science Foundation through Engineering Research Center for Revolutionizing Metallic Biomaterials (ERC-0812348) and Nanotechnology Undergraduate Education (NUE-1242139).

Author Contributions: Shalil Khanal conducted the research, analyzed the data and prepared the first draft of the manuscript. Nava P. Rijal and Udhab Adhikari contributed in particles characterization, data interpretation and manuscript preparation. Shanta Raj Bhattarai and Jagannathan Sankar contributed to writing of the manuscript and data interpretation. Narayan Bhattarai contributed to overall design, direction and supervision of the research project.

Conflicts of Interest: The authors declare no conflict of interest.

\section{References}

1. Dev, A.; Binulal, N.S.; Anitha, A.; Nair, S.V.; Furuike, T.; Tamura, H.; Jayakumar, R. Preparation of poly(lactic acid)/chitosan nanoparticles for anti-HIV drug delivery applications. Carbohydr. Polym. 2010, 80, 833-838. [CrossRef]

2. Boakye, M.A.D.; Rijal, N.P.; Adhikari, U.; Bhattarai, N. Fabrication and characterization of electrospun PCL-MgO-Keratin-Based composite nanofibers for biomedical applications. Materials 2015, 8, 4080-4095. [CrossRef]

3. Mahapatro, A.; Singh, D.K. Biodegradable nanoparticles are excellent vehicle for site directed in vivo delivery of drugs and vaccines. J. Nanobiotechnol. 2011, 9, 55. [CrossRef] [PubMed]

4. Wang, Y.; Li, P.; Kong, L. Chitosan-modified PLGA nanoparticles with versatile surface for improved drug delivery. AAPS PharmSciTech 2013, 14, 585-592. [CrossRef] [PubMed]

5. Xu, C.F.; Wang, J. Delivery systems for siRNA drug development in cancer therapy. Asian J. Pharm. Sci. 2015, 10,1-12. [CrossRef]

6. Costantino, L.; Gandolfi, F.; Bossy-Nobs, L.; Tosi, G.; Gurny, R.; Rivasi, F.; Angela Vandelli, M.; Forni, F. Nanoparticulate drug carriers based on hybrid poly(d,l-lactide-co-glycolide)-dendron structures. Biomaterials 2006, 27, 4635-4645. [CrossRef] [PubMed]

7. Zhao, K.; Zhang, Y.; Zhang, X.; Shi, C.; Wang, X.; Wang, X.; Jin, Z.; Cui, S. Chitosan-coated poly(lactic-co-glycolic) acid nanoparticles as an efficient delivery system for Newcastle disease virus DNA vaccine. Int. J. Nanomed. 2014, 9, 4609-4619. [CrossRef] [PubMed]

8. Warner, T.D.; Giuliano, F.; Vojnovic, I.; Bukasa, A.; Mitchell, J.A.; Vane, J.R. Nonsteroid drug selectivities for cyclo-oxygenase-1 rather than cyclo-oxygenase- 2 are associated with human gastrointestinal toxicity: A full in vitro analysis. Proc. Natl. Acad. Sci. USA 1999, 96, 7563-7568. [CrossRef] [PubMed]

9. Danhier, F.; Ansorena, E.; Silva, J.M.; Coco, R.; Le Breton, A.; Preat, V. PLGA-based nanoparticles: An overview of biomedical applications. J. Control. Release 2012, 161, 505-522. [CrossRef] [PubMed]

10. Sena, M.M.; Chaudhry, Z.F.; Collins, C.H.; Poppi, R.J. Direct determination of diclofenac in pharmaceutical formulations containing B vitamins by using UV spectrophotometry and partial least squares regression. J. Pharm. Biomed. Anal. 2004, 36, 743-749. [CrossRef] [PubMed]

11. Italia, J.L.; Bhatt, D.K.; Bhardwaj, V.; Tikoo, K.; Kumar, M.N. PLGA nanoparticles for oral delivery of cyclosporine: Nephrotoxicity and pharmacokinetic studies in comparison to Sandimmune Neoral. J. Control. Release 2007, 119, 197-206. [CrossRef] [PubMed]

12. Italia, J.L.; Yahya, M.M.; Singh, D.; Ravi Kumar, M.N. Biodegradable nanoparticles improve oral bioavailability of amphotericin B and show reduced nephrotoxicity compared to intravenous Fungizone. Pharm. Res. 2009, 26, 1324-1331. [CrossRef] [PubMed]

13. Bernardi, A.; Zilberstein, A.C.; Jager, E.; Campos, M.M.; Morrone, F.B.; Calixto, J.B.; Pohlmann, A.R.; Guterres, S.S.; Battastini, A.M. Effects of indomethacin-loaded nanocapsules in experimental models of inflammation in rats. Br. J. Pharmacol. 2009, 158, 1104-1111. [CrossRef] [PubMed]

14. Parveen, S.; Sahoo, S.K. Polymeric nanoparticles for cancer therapy. J. Drug Target. 2008, 16, 108-123. [CrossRef] [PubMed] 
15. Nafee, N.; Taetz, S.; Schneider, M.; Schaefer, U.F.; Lehr, C.M. Chitosan-coated PLGA nanoparticles for DNA/RNA delivery: Effect of the formulation parameters on complexation and transfection of antisense oligonucleotides. Nanomedicine 2007, 3, 173-183. [CrossRef] [PubMed]

16. Esmaeili, F.; Ghahremani, M.H.; Ostad, S.N.; Atyabi, F.; Seyedabadi, M.; Malekshahi, M.R.; Amini, M.; Dinarvand, R. Folate-receptor-targeted delivery of docetaxel nanoparticles prepared by PLGA-PEG-folate conjugate. J. Drug Target. 2008, 16, 415-423. [CrossRef] [PubMed]

17. Adhikari, U.; Rijal, N.P.; Pai, D.; Sankar, J.; Bhattarai, N. Synthesis and characterization of Chitosan-Mg-Based composite scaffolds for bone repair applications. In Proceedings of the ASME 2015 International Mechanical Engineering Congress and Exposition, Houston, TX, USA, 13-19 November 2015; American Society of Mechanical Engineers: New York, NY, USA, 2015.

18. Amoozgar, Z.; Park, J.; Lin, Q.; Yeo, Y. Low molecular-weight chitosan as a pH-sensitive stealth coating for tumor-specific drug delivery. Mol. Pharm. 2012, 9, 1262-1270. [CrossRef] [PubMed]

19. Francis Suh, J.K.; Matthew, H.W.T. Application of chitosan-based polysaccharide biomaterials in cartilage tissue engineering: A review. Biomaterials 2000, 21, 2589-2598. [CrossRef]

20. Rijal, N.P.; Adhikari, U.; Bhattarai, N. Magnesium incorporated polycaprolactone-based composite nanofibers. In Proceedings of the ASME 2015 International Mechanical Engineering Congress and Exposition, Houston, TX, USA, 13-19 November 2015; American Society of Mechanical Engineers: New York, NY, USA, 2015.

21. Chronopoulou, L.; Massimi, M.; Giardi, M.F.; Cametti, C.; Devirgiliis, L.C.; Dentini, M.; Palocci, C. Chitosan-coated PLGA nanoparticles: A sustained drug release strategy for cell cultures. Colloids Surf. B Biointerfaces 2013, 103, 310-317. [CrossRef] [PubMed]

22. Janes, K.A.; Calvo, P.; Alonso, M.J. Polysaccharide colloidal particles as delivery systems for macromolecules. Adv. Drug Deliv. Rev. 2001, 47, 83-97. [CrossRef]

23. Dodane, V.; Amin Khan, M.; Merwin, J.R. Effect of chitosan on epithelial permeability and structure. Int. J. Pharm. 1999, 182, 21-32. [CrossRef]

24. Budhian, A.; Siegel, S.J.; Winey, K.I. Controlling the in vitro release profiles for a system of haloperidol-loaded PLGA nanoparticles. Int. J. Pharm. 2008, 346, 151-159. [CrossRef] [PubMed]

25. Madhusudhan, A.; Reddy, G.B.; Venkatesham, M.; Veerabhadram, G.; Kumar, D.A.; Natarajan, S.; Yang, M.Y.; $\mathrm{Hu}$, A.; Singh, S.S. Efficient pH dependent drug delivery to target cancer cells by gold nanoparticles capped with carboxymethyl chitosan. Int. J. Mol. Sci. 2014, 15, 8216-8234. [CrossRef] [PubMed]

26. Bodmeier, R.; Chen, H.; Paeratakul, O. A Novel Approach to the Oral Delivery of Micro- or Nanoparticles. Pharm. Res. 1989, 6, 413-417. [CrossRef] [PubMed]

27. Lorenzo-Lamosa, M.L.; Remunan-Lopez, C.; Vila-Jato, J.L.; Alonso, M.J. Design of microencapsulated chitosan microspheres for colonic drug delivery. J. Control. Release 1998, 52, 109-118. [CrossRef]

28. Agnihotri, S.A.; Mallikarjuna, N.N.; Aminabhavi, T.M. Recent advances on chitosan-based micro- and nanoparticles in drug delivery. J. Control. Release 2004, 100, 5-28. [CrossRef] [PubMed]

29. Jain, R.A. The manufacturing techniques of various drug loaded biodegradable poly(lactide-co-glycolide) (PLGA) devices. Biomaterials 2000, 21, 2475-2490. [CrossRef]

30. Ludwig, A. The use of mucoadhesive polymers in ocular drug delivery. Adv. Drug Deliv. Rev. 2005, 57, 1595-1639. [CrossRef] [PubMed]

31. Gupta, H.; Aqil, M.; Khar, R.K.; Ali, A.; Bhatnagar, A.; Mittal, G. Sparfloxacin-loaded PLGA nanoparticles for sustained ocular drug delivery. Nanomedicine 2010, 6, 324-333. [CrossRef] [PubMed]

32. Shi, S.; Zhang, Z.; Luo, Z.; Yu, J.; Liang, R.; Li, X.; Chen, H. Chitosan grafted methoxy poly(ethylene glycol)-poly(epsilon-caprolactone) nanosuspension for ocular delivery of hydrophobic diclofenac. Sci. Rep. 2015, 5, 11337. [CrossRef] [PubMed]

33. Pool, H.; Quintanar, D.; Figueroa, J.D.; Mano, C.M.; Bechara, E.J.H.; Godinez, L.A.; Mendoza, S. Antioxidant effects of quercetin and catechin encapsulated into PLGA nanoparticles. J. Nanomater. 2012. [CrossRef]

34. Silva, G.S.; Oliveira, P.C.; Giordani, D.S.; de Castro, H.F. Chitosan/siloxane hybrid. polymer: Synthesis, characterization and performance as a support. for immobilizing enzyme. J. Braz. Chem. Soc. 2011, 22, 1407-1417. [CrossRef]

35. Shivakumar, H.N.; Desai, B.G.; Deshmukh, G. Design and optimization of diclofenac sodium controlled release solid dispersions by response surface methodology. Indian J. Pharm. Sci. 2008, 70, 22-30. [CrossRef] [PubMed] 
36. Tunçay, M.; Çaliş, S.; Kaş, H.S.; Ercan, M.T.; Peksoy, İ.; Hincal, A.A. Diclofenac sodium incorporated PLGA (50:50) microspheres: Formulation considerations and in vitro/in vivo evaluation. Int. J. Pharm. 2000, 195, 179-188. [CrossRef]

37. Morlock, M.; Kissel, T.; Li, Y.X.; Koll, H.; Winter, G. Erythropoietin loaded microspheres prepared from biodegradable LPLG-PEO-LPLG triblock copolymers: Protein stabilization and in vitro release properties. J. Control. Release 1998, 56, 105-115. [CrossRef]

38. McCall, R.L.; Sirianni, R.W. PLGA nanoparticles formed by single- or double-emulsion with vitamin E-TPGS. J. Vis. Exp. 2013. [CrossRef] [PubMed]

39. Mathew, A.; Fukuda, T.; Nagaoka, Y.; Hasumura, T.; Morimoto, H.; Yoshida, Y.; Maekawa, T.; Venugopal, K.; Kumar, D.S. Curcumin loaded-PLGA nanoparticles conjugated with Tet-1 peptide for potential use in Alzheimer's disease. PLoS ONE 2012, 7, e32616. [CrossRef] [PubMed]

(C) 2016 by the authors; licensee MDPI, Basel, Switzerland. This article is an open access article distributed under the terms and conditions of the Creative Commons Attribution (CC-BY) license (http://creativecommons.org/licenses/by/4.0/). 